\title{
CRESCIMENTO EM DIÂMETRO, ALTURA E VOLUME DE Hovenia dulcis NA REGIÃO SUL DE BLUMENAU, SC
}

\author{
Jackson Roberto Eleotério $^{1}$, Gabriel Corso Pellens ${ }^{2}$, Marcelo Jorge Commanduli ${ }^{3}$ \\ ${ }^{1}$ Eng. Florestal, Dr., Depto. Engenharia Florestal, FURB, Blumenau, SC, Brasil -jreleote@ furb.br \\ ${ }^{2}$ Eng. Florestal, Mestrando em Engenharia Florestal, FURB, Blumenau, SC, Brasil -gabrielpellens @ terra.com.br \\ ${ }^{3}$ Eng. Florestal, Erechim, RS, Brasil -mjcommanduli@bol.com.br \\ Recebido para publicação: 22/11/2011 - Aceito para publicação: 24/08/2012
}

\begin{abstract}
Resumo
Uva-do-japão (Hovenia dulcis Thunberg) é uma espécie difundida no Sul do Brasil, mas informações sobre o seu crescimento são raras. O objetivo deste trabalho foi avaliar, através da análise de tronco, o crescimento em diâmetro, altura e volume de Hovenia dulcisna região sul de Blumenau. Foram amostradas duas árvores e mensuradas as alturas totais, sendo retirados discos a $0,1 \mathrm{~m}, 0,7 \mathrm{~m}, 1,3 \mathrm{~m}$ e de um em um metro ao longo do comprimento da árvore, até o diâmetro mínimo de $5 \mathrm{~cm}$. As informações de diâmetro, altura e volume em função da idade foram utilizadas para calcular os incrementos correntes e médios anuais. O crescimento em diâmetro, até os 20 anos de idade, manteve-se praticamente constante, sem definição de um ponto de máximo. O crescimento em altura apresentou taxas decrescentes, com máximo incremento corrente aos cinco anos e máximo incremento médio aos 11 anos. O crescimento em volume apresentou taxas crescentes, reflexo do crescimento constante do diâmetro. $\mathrm{O}$ fator de forma artificial seguiu uma tendência de decréscimo até o décimo terceiro ano e estabilização a partir desse momento.

Palavras-chave: Análise de tronco; incremento; uva-do-japão; modelagem; regressão.
\end{abstract}

\begin{abstract}
Diameter, height and volume growth of Hovenia dulcis in south Blumenau, SC. Japanese Raisin Tree (Hoveniadulcis Thunberg) is a widely spread species in South Brazil, but information on its growing patterns is rare. The aim of this work is to evaluate, through stem analysis, the diameter, height and volume growth of Hovenia dulcis in South Blumenau. Two trees were sampled, and their total height was measured. Discs were taken at $0.1 \mathrm{~m}, 0.7 \mathrm{~m}, 1.3 \mathrm{~m}$. and at one-meter intervals along the length of the tree, until the minimum of $5 \mathrm{~cm}$ of diameter. The information on diameter, height and volume in function of the age were used to calculate the current and the mean annual increment. The diameter growth remained practically constant, until 20 years, without any definition of a peak of increment. The height growth presented decreasing rates, with a peak of current increment occurring in the fifth year and a peak of average annual increment in the eleventh year. The volume growthpresented increasing rates as result of the constant diameter growth. The artificial form factor followed a decreasing tendency until the thirteenth year and established since that moment.

Keywords: Stem analysis; increment; Japanese Raisin tree; modeling; regression.
\end{abstract}

\section{INTRODUÇÃO}

Uva-do-japão (Hoven adulcis Thunberg) pertence à família Rhamnaceae e ocorre naturalmente na China, Japão e Coreia. No Sul do Brasil, é largamente difundida pelo cultivo em pequenos talhões ou de forma isolada em propriedades rurais ao longo da bacia do rio Uruguai, nos estados de Santa Catarina e Rio Grande do Sul e no sudoeste do Paraná (CARVALHO, 1994).

A espécie é cultivada principalmente com a finalidade de planta ornamental, quebra-vento e para produção de madeira para uso energético e construção civil. Seu crescimento é bastante variável no Brasil, atingindo $30 \mathrm{~m}^{3} \cdot \mathrm{ha}^{-1} \cdot$ ano $^{-1}$ (CARVALHO, 1994). Rigatto et al.(2001) afirmam que a altura varia de 10 a $15 \mathrm{~m}$, podendo atingir $25 \mathrm{~m}$. Já o diâmetro à altura do peito (DAP) varia de $20 \mathrm{a} 40 \mathrm{~cm}$, podendo alcançar $50 \mathrm{~cm}$. 
A disponibilidade de informações sobre crescimento dessa espécie é bastante limitada, merecendo destaque a classificação de sítios apresentada por Selle (2009).

A madeira dessa espécie é classificada como moderadamente pesada, com massa específica entre 500 e $720 \mathrm{~kg} / \mathrm{m}^{3}$. Algumas propriedades organolépticas, como cor do cerne, de amarelo a castanho-escuro ou vermelho, brilho opaco a mediano, textura fina e grã direita permitem utilizar a espécie na indústria moveleira (CARVALHO, 1994).

Assim como na madeira de outras espécies caducifólias, na madeira de Hovenia dulcis existe a formação de anéis anuais de crescimento demarcados por uma transição abrupta na frequência e no tamanho dos vasos. Essa característica foi confirmada por Chagas (2009) e utilizada para dendrocronologia.

Ainda acerca da demarcação de anéis de crescimentos em uva-do-japão, trabalhos realizados por Suzuki (1982) afirmam que esses anéis de crescimento são distintos pela porosidade dos anéis.

A existência de anéis anuais de crescimento delimitáveis possibilita a realização da análise de tronco. A técnica da análise de tronco permite obter informações sobre o crescimento em diâmetro, altura e volume ocorrido em anos anteriores. Finger (1992) classifica a análise de tronco em completa, quando são retirados discos ao longo da altura da árvore, e parcial, quando são obtidos apenas rolos de incremento.

Com o avanço das técnicas de análise de imagens, a análise de tronco tem sido facilitada pela digitalização dos discos, identificação e medição dos anéis, com o auxílio de softwares específicos. Um exemplo dessa possibilidade é apresentado por Rosot (2003), que acrescenta como vantagem a possibilidade de analisar o crescimento a partir de discos saturados de umidade, próximos da condição natural das árvores.

O objetivo deste trabalho foi demonstrar, através da análise de tronco, que a espécie em questão apresentou rápido crescimento em diâmetro, altura e volume no Médio Vale do Itajaí.

\section{MATERIAL E MÉTODOS}

\section{Localização do plantio e árvores amostradas}

Os dados foram coletados de árvores de ocorrência espontânea no Parque Ecológico Spitzkoft $\left(27^{\circ} 01^{\prime} \mathrm{S}, 49^{\circ} 07^{\prime} \mathrm{W}\right)$, município de Blumenau, SC.

Segundo a classificação climática de Köppen, a região do Vale do Itajaí enquadra-se como clima $\mathrm{Cfa}$, ou seja, clima subtropical constantemente úmido, sem estação seca, com verão quente (temperatura média do mês mais quente $>22^{\circ} \mathrm{C}$ ). A temperatura média anual varia de 19,1 a $20,0^{\circ} \mathrm{C}$, com temperatura média das máximas variando de 26,0 a $27,6{ }^{\circ} \mathrm{C}$ e a média das mínimas variando entre 15,4 e $16,8{ }^{\circ} \mathrm{C}$. A precipitação pluviométrica total anual pode variar de 1.430 a $1.908 \mathrm{~mm}$, distribuída em 156 a 185 dias de chuva (THOME; EMPRESA DE PESQUISA AGROPECUÁRIA E EXTENSÃO RURAL DE SANTA CATARINA (EPAGRI), 1999).

O solo da região é classificado como Ra1, ou seja, associação entre solos litólico sálicos A moderado, textura argilosa, fase pedregosa com substrato formado por rochas do Complexo Metamórfico Brusque e Cambissolo álico Tb A moderado, textura argilosa, relevo montanhoso e escarpado (EMPRESA BRASILEIRA DE PESQUISA AGROPECUÁRIA (EMBRAPA), 1998;CENTRO DE INFORMAÇÕES DE RECURSOS AMBIENTAIS DE SANTA CATARINA (CIRAM), 2002).

Foram amostradas duas árvores que cresciam em um talhão de Cunninghamia lanceolata. Após o abate das árvores, foi medida a altura total e retirados discos a $0,1 \mathrm{~m}, 0,7 \mathrm{~m}, 1,3 \mathrm{~m}$ e de um em um metro ao longo do comprimento da árvore, até o diâmetro mínimo de $5 \mathrm{~cm}$.

\section{Marcação dos anéis de crescimento}

Em cada um dos discos, ainda verdes, foi localizado o maior raio e, a partir deste, marcadas duas linhas diametrais, formando um ângulo de $45^{\circ} \mathrm{com}$ o raio referido. Para facilitar a identificação e medição dos anéis anuais de crescimento, a superfície dos discos sobre as linhas diametrais foi aplainada com plaina elétrica portátil.

Utilizou-se um método digital para medição de anéis de crescimento, iniciando com a digitalização dos discos através de scanner. As imagens geradas foram exportadas para um programa Computer Aided Design (CAD), no qual os anéis anuais foram demarcados e medidos (Figura 1). A largura média de quatro raios foi considerada como a largura do anel de crescimento. Montado o perfil da árvore, as alturas totais em cada idade foram determinadas com base no método do paralelismo ao anel imediatamente anterior, como apresentado em Rosot et al.(2003). 
Os volumes dos segmentos entre os discos foram calculados pelo método de Smalian, e a sua somatória forneceu o volume total sem casca para cada idade.

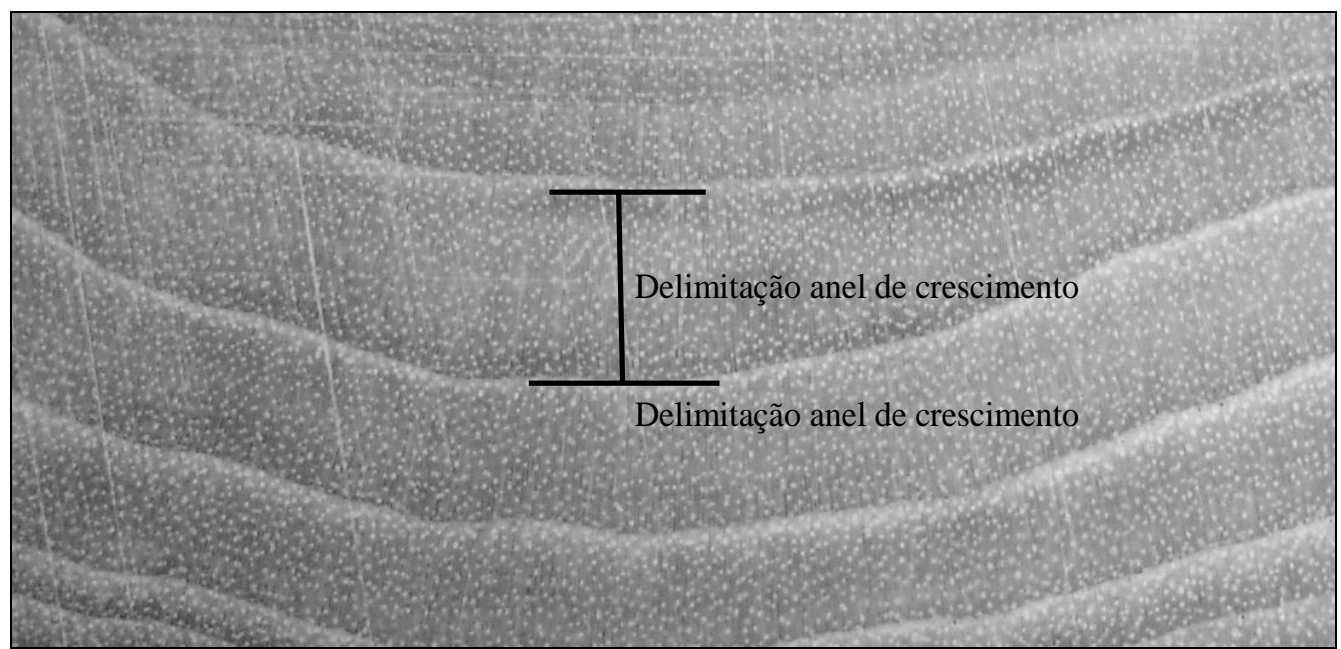

Figura 1. Delimitação dos anéis de crescimento em madeira de Hovenia dulcis.

Figure 1. Limitation of growth rings in wood of Hovenia dulcis wood.

\section{Crescimento e forma das árvores}

As informações de diâmetro, altura e volume em função da idade foram utilizadas para o cálculo dos incrementos correntes e médios anuais. Da mesma forma, foram utilizadas para ajustar funções de crescimento para essas variáveis. As funções avaliadas estão apresentadas na tabela 1.

Tabela 1. Funções de crescimento ajustadas para estimar diâmetro, altura e volume (variável y) em função da idade.

Table 1. Growth equations adjusted to estimate diameter, height and volume (variable y) in function of the age.

\begin{tabular}{l|c|c}
\hline $\mathbf{N}^{\circ}$ da função & Função & Autor \\
\hline 1 & $\ln \mathrm{y}=\mathrm{b} 0+\mathrm{b} 1 * 1 / \mathrm{t}$ & Schumacker \\
\hline 2 & $\ln \mathrm{y}=\mathrm{b} 0+\mathrm{b} \cdot \ln \mathrm{t}+\mathrm{b} 2 \cdot \ln ^{2} \mathrm{t}$ & Backman \\
\hline 3 & $\ln \mathrm{y}=\mathrm{b} 1 \cdot \ln \mathrm{t}+\mathrm{b} 2 \cdot \ln ^{2} \mathrm{t}$ & Prodan \\
\hline 4 & $\mathrm{y}=\frac{\mathrm{t}^{2}}{\mathrm{~b} 0+\mathrm{b} 1 . \mathrm{t}+\mathrm{b} 2 \cdot \mathrm{t}^{2}}$ & Prodan modificado \\
\hline 5 & $\mathrm{y}=\frac{\mathrm{t}^{2}}{\mathrm{~b} 1 . \mathrm{t}+\mathrm{b} 2 . \mathrm{t}^{2}}$ & Horel \\
\hline 6 & $\ln \mathrm{y}=\mathrm{b} 0+\mathrm{b} 1 \cdot 1 / \mathrm{t}+\mathrm{b} 2 \cdot \ln \mathrm{t}$ & Moissev \\
\hline 7 & $\ln \mathrm{y}=\mathrm{b} 0+\mathrm{b} 1 . \mathrm{t}+\mathrm{b} 2 \cdot \mathrm{t}^{2}+\mathrm{b} 3 \cdot \mathrm{t}^{3}$ & Gram \\
\hline 8 & $\ln \mathrm{y}=\mathrm{b} 0+\mathrm{b} 11 \cdot \ln \mathrm{t}+\mathrm{b} 2 . \mathrm{t}$ & \\
\hline
\end{tabular}

A partir desses dados, foram testados diferentes modelos de ajuste para variáveis independentes (diâmetro, altura e volume). Para cada um dos modelos, foi realizada regressão linear, definindo variáveis de ajuste de equação, coeficiente de determinação ajustado $\left(\mathrm{R}^{2}\right)$, erro padrão da estimativa (Sxy\%), coeficiente de variação (CV\%) e F crítico.

Levando em consideração os critérios gerados pela regressão linear, estabeleceu-se um escore para cada um desses critérios, com a finalidade de avaliar qual o melhor modelo ajustado para cada uma das variáveis mensuradas. Ao final, com os valores determinados, eles foram ponderados, o que auxiliou na avaliação do melhor modelo testado. 
Em trabalhos realizados por Thomas et al. (2006), esse critério de seleção leva em consideração todas as variáveis estatísticas anteriormente descritas e por isso sintetiza os resultados, facilitando o processo de seleção do melhor modelo matemático.

O valor ponderado foi determinado atribuindo-se valores ou pesos aos parâmetros estatísticos. As estatísticas foram ordenadas de acordo com a sua eficiência, sendo atribuído peso 1 para a equação mais eficiente, 2 para a segunda e assim sucessivamente (ranking), conforme metodologia adotada por Thomas et al. (2006).A determinação e o estabelecimento desse escore são em função do maior coeficiente de determinação $\left(\mathrm{R}^{2}\right)$, menor erro padrão da estimativa $\left(\mathrm{S}_{\mathrm{xy}} \%\right)$, menor coeficiente de variação (CV) e maior F crítico.

Após essa classificação individual, efetuou-se o somatório da pontuação para cada modelo matemático, sendo que a equação de volume que recebeu a menor soma foi recomendada como mais adequada para uso.

\section{RESULTADOS E DISCUSSÃO}

No momento do abate, a árvore 1 estava com 20 anos, altura total de $22,6 \mathrm{~m}$ e DAP de $23,2 \mathrm{~cm}$, e a árvore 2 estava com 19 anos, DAP de 24,2 cm e altura total de 21,4 m. O percentual médio de casca era de $8 \%$.

O processamento digital das imagens resultou na análise de tronco para Hovenia dulcis, na qual os valores de crescimento, incremento médio anual e incremento corrente para cada um dos anéis de crescimento foram obtidos.

Os resultados estatísticos do ajuste das equações testadas para estimar o crescimento em diâmetro e volume comercial em função da idade encontram-se apresentados na tabela 2.

Tabela 2. Resultados estatísticos do ajuste das equações testadas para estimar diâmetro, altura e volume em função da idade.

Table 2. Statistical results for the fitted equations to estimate diameter, height and volume in function of the age.

\begin{tabular}{|c|c|c|c|c|c|c|c|c|c|c|}
\hline $\begin{array}{l}\text { Variável } \\
\text { dependente }\end{array}$ & Modelo & $\mathbf{R}^{2}$ & Sxy\% & CV\% & $\mathbf{F}$ & $\mathbf{R}^{2}$ & Sxy \% & CV\% & $\mathbf{F}$ & Valor ponderado \\
\hline \multirow{8}{*}{ Diâmetro } & 1 & 0,796 & 3,13 & 28,60 & 149,3 & 8 & 1 & 8 & 8 & 25 \\
\hline & 2 & 0,984 & 4,81 & 7,72 & 1203,3 & 2 & 4 & 6 & 4 & 16 \\
\hline & 3 & 0,971 & 4,76 & 7,67 & 9855,4 & 5 & 2 & 4 & 1 & 12 \\
\hline & 4 & 0,967 & 9,84 & 7,48 & 565,8 & 6 & 8 & 1 & 6 & 21 \\
\hline & 5 & 0,966 & 9,82 & 7,90 & 2590,6 & 7 & 7 & 7 & 2 & 23 \\
\hline & 6 & 0,984 & 4,81 & 7,67 & 1203,5 & 1 & 3 & 3 & 3 & 10 \\
\hline & 7 & 0,976 & 5,74 & 7,56 & 560,2 & 4 & 6 & 2 & 7 & 19 \\
\hline & 8 & 0,984 & 4,83 & 7,68 & 1193,3 & 3 & 5 & 5 & 5 & 18 \\
\hline \multirow{8}{*}{ Altura } & 1 & 0,808 & 16,92 & 27,04 & 106,5 & 8 & 8 & 8 & 8 & 32 \\
\hline & 2 & 0,976 & 5,94 & 5,89 & 783,2 & 3 & 4 & 3 & 5 & 15 \\
\hline & 3 & 0,970 & 5,87 & 5,93 & 6470,9 & 5 & 2 & 4 & 1 & 12 \\
\hline & 4 & 0,982 & 7,27 & 5,54 & 1035,7 & 1 & 6 & 1 & 3 & 11 \\
\hline & 5 & 0,968 & 7,80 & 6,11 & 4101,3 & 6 & 7 & 6 & 2 & 21 \\
\hline & 6 & 0,976 & 5,94 & 5,95 & 783,1 & 4 & 5 & 5 & 6 & 20 \\
\hline & 7 & 0,966 & 1,07 & 6,34 & 356,6 & 7 & 1 & 7 & 7 & 22 \\
\hline & 8 & 0,976 & 5,93 & 5,75 & 785,9 & 2 & 3 & 2 & 4 & 11 \\
\hline \multirow{8}{*}{ Volume } & 1 & 0,976 & $-11,25$ & 12,63 & 1567,6 & 4 & 3 & 2 & 1 & 10 \\
\hline & 2 & 0,977 & $-11,05$ & 16,49 & 813,7 & 3 & 4 & 4 & 3 & 14 \\
\hline & 3 & 0,622 & $-74,00$ & 127,13 & 35,6 & 6 & 1 & 7 & 6 & 20 \\
\hline & 4 & 0,596 & 78,31 & 59,64 & 29,0 & 7 & 7 & 5 & 7 & 26 \\
\hline & 5 & 0,197 & 140,45 & 189,14 & 6,0 & 8 & 8 & 8 & 8 & 32 \\
\hline & 6 & 0,978 & $-10,84$ & 16,14 & 846,4 & 1 & 5 & 3 & 2 & 11 \\
\hline & 7 & 0,978 & $-10,84$ & 11,73 & 564,1 & 2 & 6 & 1 & 5 & 14 \\
\hline & 8 & 0,976 & $-11,27$ & 100,00 & 781,3 & 5 & 2 & 6 & 4 & 17 \\
\hline
\end{tabular}


A partir da compilação dos dados gerados através da regressão linear e com o valor ponderado para cada uma das variáveis em questão, foram determinados os melhores modelos de estimativa para cada uma das variáveis independentes analisadas.

Ao analisar os resultados contidos na tabela 2, à exceção do modelo 1, todos os demais modelos para estimar o diâmetro em função da idade apresentaram resultados que podem ser considerados satisfatórios. Entre os modelos analisados para diâmetro, o que obteve menor valor ponderado, e consequentemente melhor desempenho, foi o modelo seis. Os coeficientes desse modelo são $b 0=-0,0946$, $\mathrm{b} 1=0,1247$ e b2 $=1,1153$.

Ainda acerca da variável diâmetro, pode-se concluir que os valores máximos obtidos para incremento médio anual (IMA) foi de $1,29 \mathrm{~cm}$, obtido na idade de 20 anos. Já o incremento corrente anual (ICA) tem seu ponto máximo também na idade de 20 anos, com 1,43 cm, apesar de o crescimento manterse aproximadamente constante no período analisado.

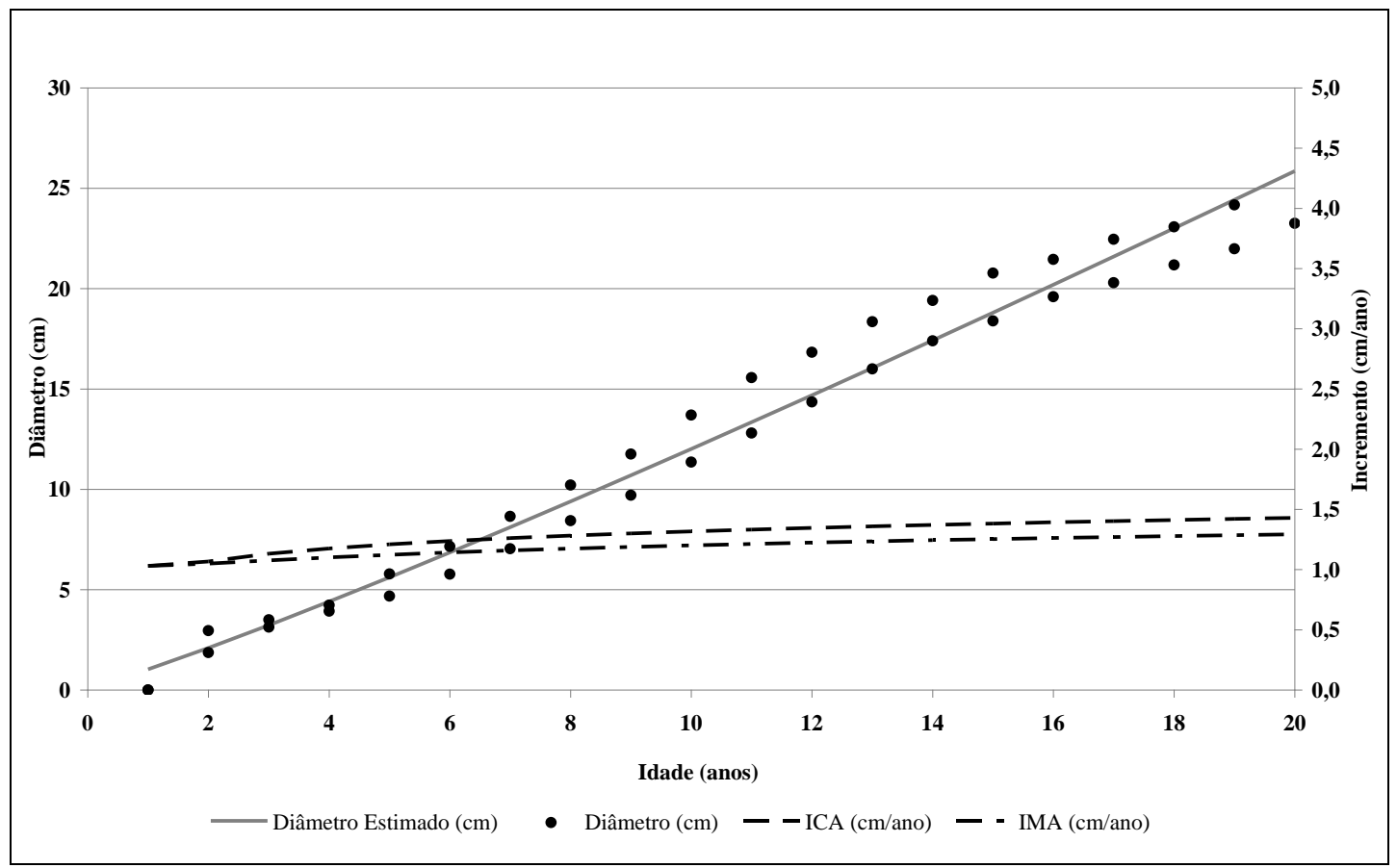

Figura 2. Diâmetro e incrementos corrente e médio em função da idade.

Figure 2. Diameter, current and mean increment in function of the age.

Analisando os modelos testados para a variável altura, conclui-se que o modelo quatro obteve melhor desempenho e consequentemente menor valor ponderado. Os coeficientes desse modelo são $\mathrm{b} 0=0,8530 ; \mathrm{b} 1=0,6546 \mathrm{e}$ b2 $=0,0073$. Os valores estimados por esse modelo são praticamente paralelos aos obtidos por Selle (2009) para a altura dominante das árvores em um sítio com índice de sítio 24.

Os pontos de máximo para o incremento corrente anual (ICA) ocorreu aos 5 anos, apresentando $1,343 \mathrm{~m}$. Já o incremento médio anual (IMA) teve ponto de máximo no $11^{\circ}$ ano, com $1,231 \mathrm{~m}$.

A última variável avaliada, segundo modelos de predição de crescimento, foi o volume. O modelo um obteve melhor performance quando comparado aos demais, com coeficientes b0 $=-9,3075$ e b1 $=2,9077$. As derivadas desse modelo não permitiram identificar os pontos de máximo incremento corrente e médio.

Os dados reais de diâmetro, altura e volume permitiram determinar a variação do fator de forma artificial com a idade. Como ocorre em outras espécies, o fator de forma tende a diminuir com a idade ou com o crescimento da árvore (Figura 5).

Observa-se que ocorre uma estabilização nos fatores de forma analisados nas duas árvores, a partir do $13^{\circ}$ ano. 


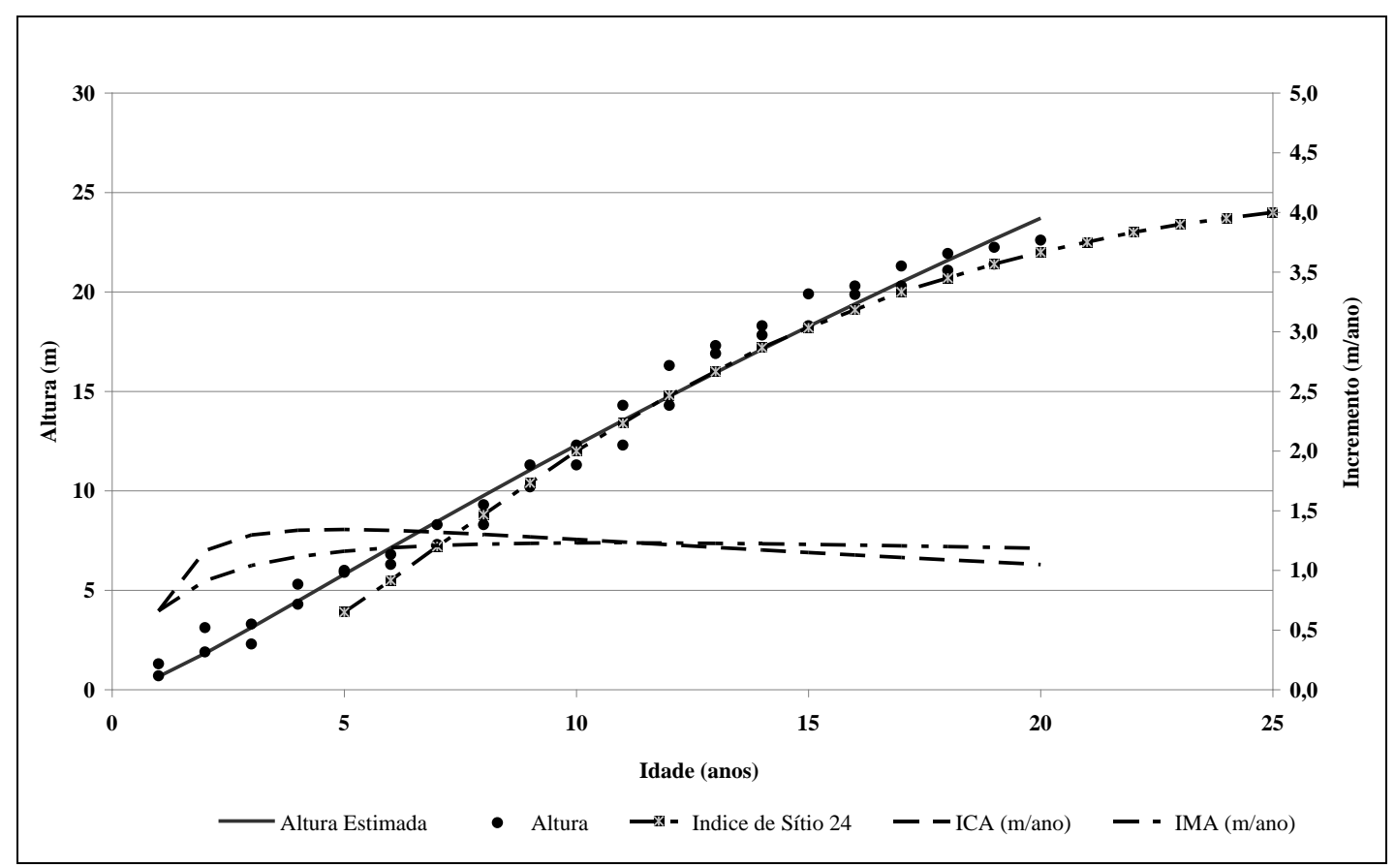

Figura 3. Altura e incrementos médio e corrente em função da idade.

Figure 3. Height, current and mean increment in function of the age.

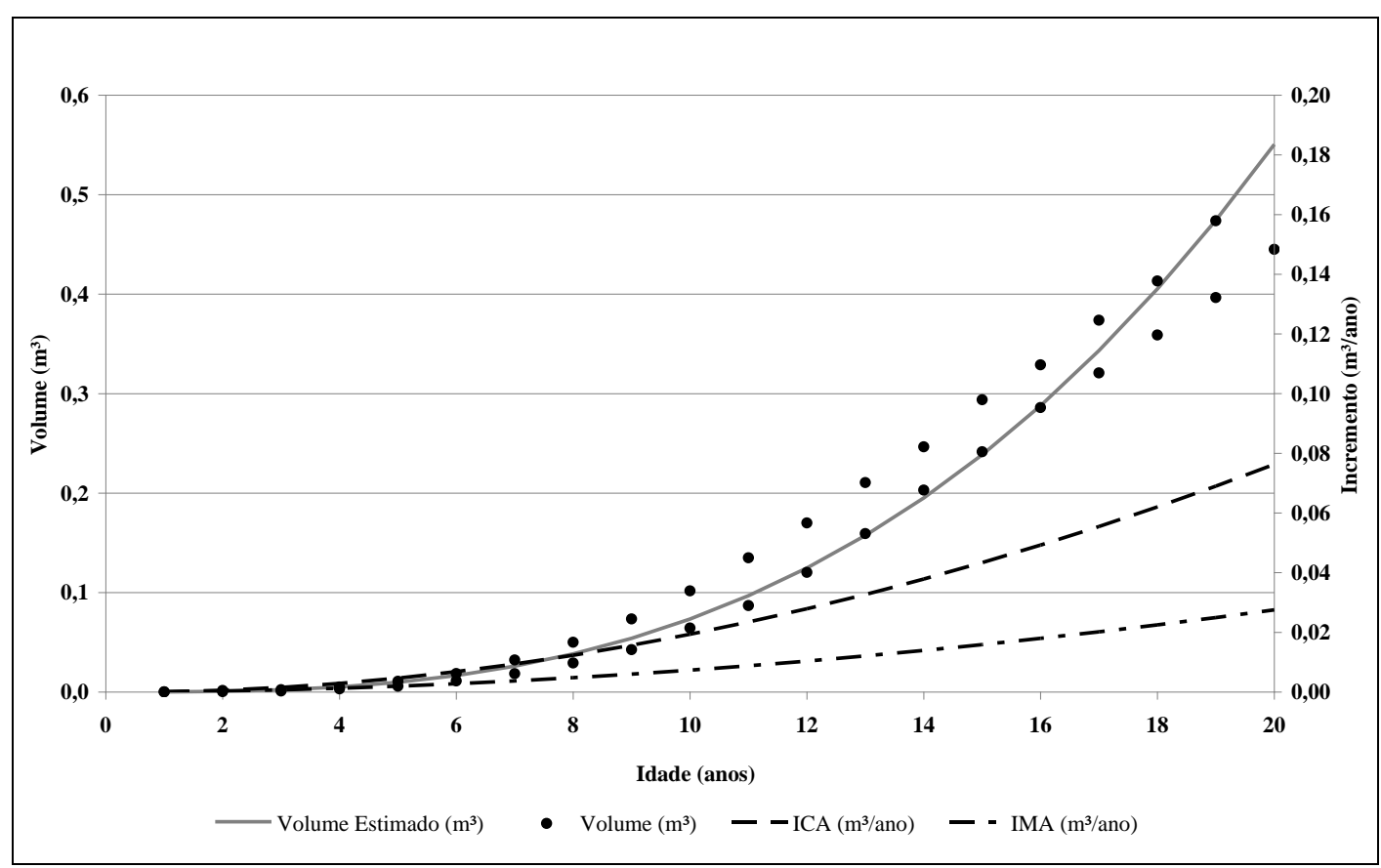

Figura 4. Volume e incrementos corrente e médio em função da idade.

Figure 4. Volume and current and mean increments in function of the age. 


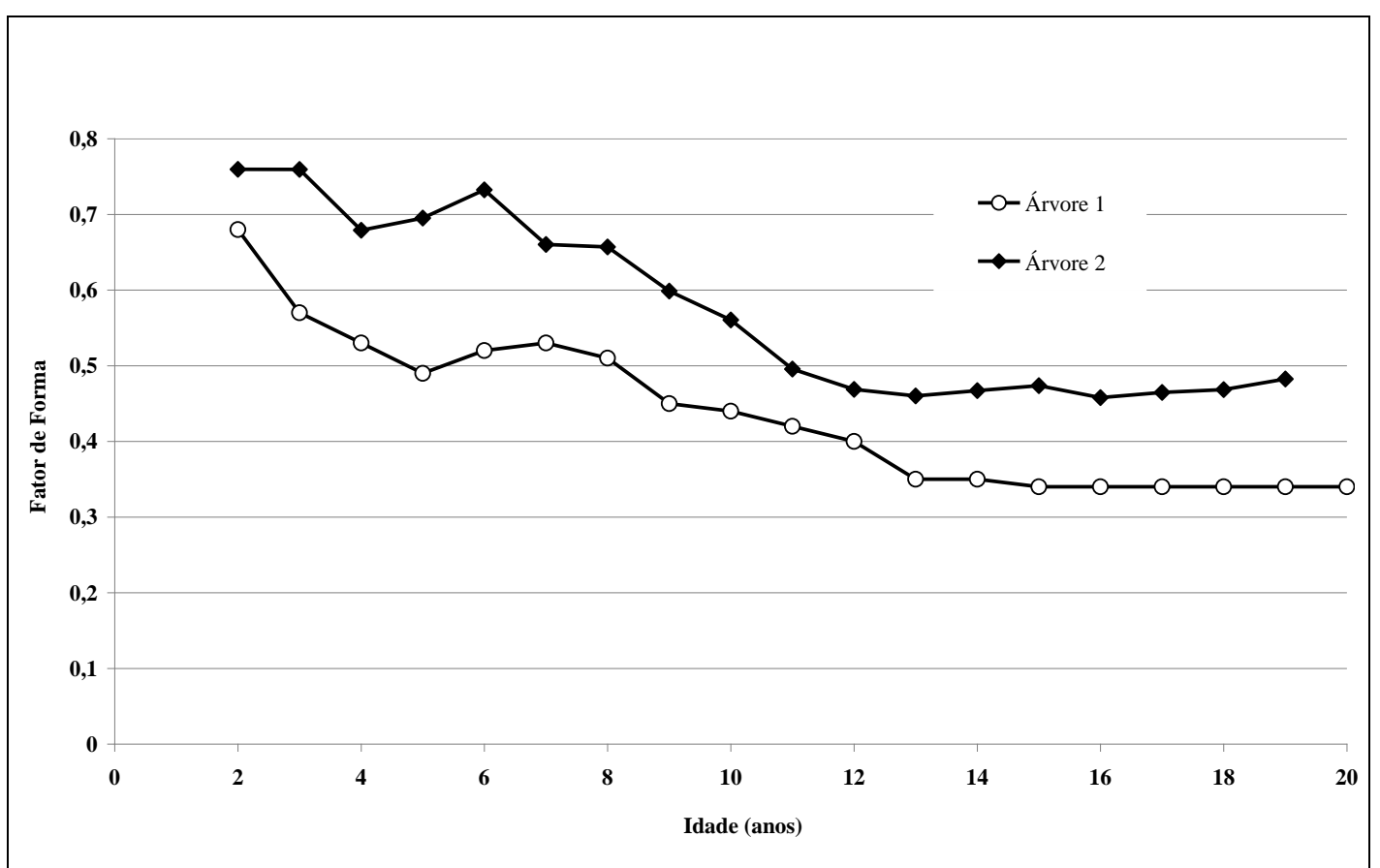

Figura 5. Fator de forma artificial em função da idade.

Figure 5. Artificial form factor in function of the age.

\section{CONCLUSÕES}

- A demarcação de anéis anuais de crescimento permitiu, através da análise de tronco, determinar o crescimento em diâmetro, altura e volume de Hovenia dulcis.

- O crescimento em diâmetro, até os 20 anos de idade, manteve-se praticamente constante, sem a definição de um ponto de máximo incremento.

- O crescimento em altura apresentou taxas decrescentes, com máximo incremento corrente aos cinco anos e máximo incremento médio aos 11 anos.

- O crescimento em volume apresentou, no período considerado, taxas crescentes, reflexo do crescimento praticamente constante do diâmetro.

- O fator de forma artificial seguiu uma tendência de decréscimo até o décimo terceiro ano e estabilização a partir desse momento.

\section{REFERÊNCIAS}

CARVALHO, P. E. R. Ecologia, silvicultura e usos da uva-do-japão (Hovenia dulcis Thunberg). Colombo: EMBRAPA-CNPF, 1994. 24p. (EMBRAPA-CNPF. Circular Técnica, 23).

CENTRO DE INFORMAÇÕES DE RECURSOS AMBIENTAIS DE SANTA CATARINA (CIRAM). Mapa de solos: Unidade de Planejamento Regional Litoral Norte Catarinense UPR 6. Florianópolis, 2002. 85,5 x 65,5 cm. Escala 1:250.000, 2002.

CHAGAS, M. P. Caracterização dos anéis de crescimento e dendrocronologia de árvores de Grevillea robusta A. Cunn, Hovenia dulcis Thunb., Persea americana Mill., Tabebuia pentaphylla Hemsl. E Terminalia catappa L. nos municípios de Piracicaba e Paulínia, SP. 113 f. Dissertação (Mestrado em Recursos Florestais) - Escola Superior de Agricultura "Luiz de Queiroz"- USP, Piracicaba, 2009. 
EMPRESA BRASILEIRA DE PESQUISA AGROPECUÁRIA (EMBRAPA). Centro Nacional de Pesquisa de Solos. Levantamento de reconhecimento dos solos do Estado de Santa Catarina. Rio de Janeiro, 1998. (Embrapa-CNPS. Boletim de Pesquisa, 6). CD-ROM.

FINGER, C. A. G. Fundamentos de biometria florestal. Santa Maria: CEPEF/FATEC/UFSM, 1992. $269 \mathrm{p}$.

RIGATTO, P. A.; PEREIRA, J. C. D.; MATTOS, P. P.; SCHAITZA, E. G. Características físicas, químicas e anatômicas da madeira de Hovenia dulcis. Colombo: EMBRAPA Florestas, 2001. 4 p. (Comunicado Técnico).

ROSOT, M. A. D.; FIGUEIREDO FILHO, A.; DISPERATI, A. A.; EMERENCIANO, D. B. Análise de tronco digital: uma nova metodologia para a medição de anéis de crescimento. Floresta, Curitiba, v. 33, n. 3, p. $235-255,2003$.

SELLE, G. L. Guias de densidade e índices de sítios para Hovenia dulcis Thunberg na Região Central do Estado Rio Grande do Sul, Brasil. 97 f. Tese (Doutorado em Engenharia Florestal) Universidade Federal de Santa Maria, Santa Maria, 2009.

SUZUKI, M. Some Fossil Woods from the Palaeocene of Northern Kyushu, II. Botanic Magazine. Tóquio, n. 95.p. 281 - 294, 1982.

THOMAS, C.; ANDRADE, C. M.; SCHNEIDER, P. R.; FINGER, C. A. G. Comparação de equações volumétricas ajustadas com dados de cubagem e análise de tronco. Ciência Florestal, Santa Maria, v. 16, n. 3, p. 319 - 327, 2006.

THOME, V. M. R.; EMPRESA DE PESQUISA AGROPECUÁRIA E EXTENSÃO RURAL DE SANTA CATARINA (EPAGRI). Zoneamento agroecológico e socioeconômico do Estado de Santa Catarina. Florianópolis: EPAGRI, 1999. 1 CD-ROM. (Epagri, 01/99). Versão preliminar. 